\title{
Protamine dissociation before decondensation of sperm nuclei during in vitro fertilization of pig oocytes
}

\author{
A. Shimada ${ }^{1 *}$, K. Kikuchi $^{1+}$, J. Noguchi ${ }^{1}$, K. Akama ${ }^{2,3}$, M. Nakano ${ }^{2}$ and H. Kaneko ${ }^{1}$ \\ ${ }^{1}$ Department of Genetic Resources II, National Institute of Agrobiological Resources, Tsukuba, Ibaraki 305-8602, Japan; and \\ ${ }^{2}$ Department of Chemistry, Faculty of Science and ${ }^{3}$ Graduate School of Science and Technology, Chiba University, Chiba 263-8522, Japan
}

\begin{abstract}
The correlation between morphological changes and the dynamics of protamine in boar sperm chromatin during in vitro fertilization of pig oocytes matured in vitro was assessed. For this purpose, protamine was purified from boar sperm nuclei and an antiserum against protamine was developed. After affinity purification, the antiserum reacted exclusively with boar protamine during western blotting, showing no crossreactivity with core histones. Immunohistochemical evaluation revealed that only fully developed spermatid nuclei in boar testes stained strongly with the antiserum. When pig oocytes matured in vitro were fertilized in vitro, sperm penetration was observed in $37 \%$ of oocytes at $2 \mathrm{~h}$ after insemination and the penetration rate increased to $99 \%$ by $5 \mathrm{~h}$ after insemination, accompanied by an increase in polyspermic penetration. Paraffin wax sections of the inseminated oocytes were examined by immunohistochemical analysis with the antiserum. The proportion of condensed sperm nuclei that reacted with the antiserum was $87 \%$ of the sperm nuclei that penetrated by $2 \mathrm{~h}$ after insemination, and this decreased to 20 and $13 \%$ at 3 and $5 \mathrm{~h}$ after insemination, respectively. However, none of the decondensing sperm nuclei or male pronuclei reacted with the antiserum during the entire insemination period. These results indicate that a specific antiserum against boar protamine can be raised and, using this serum, it has been demonstrated that protamine is dissociated from boar sperm nuclei before decondensation during in vitro fertilization.
\end{abstract}

\section{Introduction}

Highly condensed nuclei of mature spermatozoa from most animals contain a specific set of strongly basic DNA-binding proteins, which are termed protamines or sperm-specific nuclear proteins, depending on their biochemical properties. Of such proteins, small arginine-rich proteins are categorized as protamines. Protamines directly replace histones in most species, with the exception of mammals. In mammals, nucleosomal histones are replaced transiently by transition proteins, and finally by protamines (Poccia, 1986). Mammalian sperm protamines differ from those of other species, such as fish, amphibians and fowl, with respect to the presence of cysteine (reviewed by Poccia, 1986; Zirkin et al., 1989). The fundamental structure of sperm chromatin is not understood completely. However, it has been shown that mammalian sperm protamines have many disulphide (S-S) bonds. During epididymal maturation of spermatozoa, the thiol $(\mathrm{SH})$ groups of the cysteine residues are progressively oxidized and S-S bonds are formed (Calvin et al., 1973; Marushige and Marushige, 1975; Akama et al., 1989). The

*Present address: Department of Animal Reproduction, National Institute of Animal Industry, Tsukuba, Ibaraki 305-0901, Japan.

${ }^{\dagger}$ Correspondence.

Received 11 February 2000. formation of the disulphide linkages makes sperm nuclei tight, or compact, and resistant to detergents (Calvin and Bedford, 1971; Bedford and Calvin, 1974), trypsin (Meistrich et al., 1976) and micrococcal nuclease (Tobita et al., 1984). Mature spermatozoa are transcriptionally inactive (Katagiri and Ohsumi, 1994).

After penetration of mature spermatozoa into oocytes, the chromatin of the sperm nuclei is remodelled into a typical somatic state, participating in subsequent chromosomal activities such as decondensation of sperm nuclei and formation of male pronuclei for the completion of fertilization. Investigation of the events of chromosomal remodelling in penetrating sperm nuclei would help to elucidate the molecular changes that occur at this early stage of fertilization and may contribute to the success of technologies for assisted reproduction in mammals, such as intracytoplasmic sperm injection. Yanagimachi (1994) suggested that chromosomal remodelling requires two steps: firstly, reduction of S-S to S-H and, secondly, replacement of protamines by histones. The ability for S-S reduction in ooplasm may depend on the concentration of free thiol glutathione (Zirkin et al., 1989) as a result of cytoplasmic maturation (Yoshida, 1993; Yoshida et al., 1993). However, the mechanism of the replacement of protamines by histones is still unclear. Perreault (1992) concluded that, in mammalian 
fertilized oocytes, removal of protamine during fertilization occurs as the sperm nucleus decondenses. However, in mice, Nonchev and Tsanev (1990) reported that protamine loss occurs as histones appear in the well-developed male pronucleus. Thus, it remains unclear exactly when protamine dissociation occurs during fertilization.

Experiments to detect protamine in penetrating sperm nuclei have been carried out using radiolabelled protamine (Ecklund and Levine, 1975; Kopecny and Pavlok, 1975) or antibodies raised against sperm basic nuclear protein (Rodman et al., 1981) or protamine (Nonchev and Tsanev, 1990). An immunohistochemical approach with a wellcharacterized antibody seems to be useful for analysing the dynamics of proteins during fertilization. In some mammals, protamine has been detected during spermatogenesis by using immunofluorescence or immunoperoxidase techniques (Rodman et al., 1979; Roux et al., 1988); however, studies during fertilization have been performed only in mice (Rodman et al., 1981; Nonchev and Tsanev, 1990). Although the amino acid sequence of protamines is conserved among mammals (Tobita et al., 1983), the specificity of the antibody against protamine must be demonstrated (Stanker et al., 1993). Thus, protamine-specific immunological studies provide a new approach that could be useful for investigating the remodelling of sperm chromatin during fertilization.

In the present study, protamine was purified from boar sperm nuclei and an antiserum against protamine was developed. This antiserum was used to investigate the correlation between the morphological changes and the dynamics of protamine in boar sperm chromatin during in vitro fertilization (IVF) of pig oocytes matured in vitro.

\section{Materials and Methods}

\section{Purification of boar reduced protamine}

Sperm nuclei. Ejaculated semen from a crossbred boar was filtered with cotton gauze and stored at $-20^{\circ} \mathrm{C}$ until used for purification. Isolation of sperm nuclei was performed at $4^{\circ} \mathrm{C}$ as described by Tobita et al. (1982), with slight modifications. After thawing, semen $(300 \mathrm{ml})$ was centrifuged at $4000 \mathrm{~g}$ for $20 \mathrm{~min}$. The sediment was washed three times with $125 \mathrm{ml}$ of a buffer solution containing 0.05 mol Tris- $\mathrm{HCl} \mathrm{l}^{-1}(\mathrm{pH} 8.3)$ and $0.14 \mathrm{~mol} \mathrm{NaCl} \mathrm{l}^{-1}$. The washed sediment was suspended in $150 \mathrm{ml}$ of a buffer containing 0.05 mol Tris- $\mathrm{HCl}^{-1}(\mathrm{pH}$ 8.0)

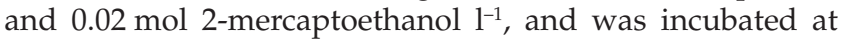
$25^{\circ} \mathrm{C}$ for $30 \mathrm{~min}$. After incubation, the suspension was centrifuged at $4000 \mathrm{~g}$ for $20 \mathrm{~min}$ at $25^{\circ} \mathrm{C}$. The resulting sediment was washed four times with $75 \mathrm{ml} 0.05 \mathrm{~mol}$ Tris- $\mathrm{HCl} \mathrm{l}^{-1}$ ( $\left.\mathrm{pH} \quad 7.0\right)$ supplemented with $1 \% \quad(\mathrm{w} / \mathrm{v})$ cetyltrimethylammonium bromide and washed three times with $75 \mathrm{ml} \mathrm{1 \% (w/v)} \mathrm{citric} \mathrm{acid.} \mathrm{The} \mathrm{washed} \mathrm{sediment}$ containing sperm nuclei was dispersed in $100 \mathrm{ml}$ buffer containing $1.62 \mathrm{~mol}$ sucrose $\mathrm{1}^{-1}$ and $0.01 \mathrm{~mol}$ Tris- $\mathrm{HCl} \mathrm{l^{-1 }}$ ( $\mathrm{pH}$ 7.4), and was recovered by centrifugation at $4000 \mathrm{~g}$ for $40 \mathrm{~min}$. This process was repeated several times until the supernatant was reasonably clear and pure nuclei were obtained.
Purification of protamine. The purified sperm nuclei were suspended in $200 \mathrm{ml}$ buffer containing $2 \mathrm{~mol} \mathrm{NaCl}{ }^{-1}, 4 \mathrm{~mol}$ urea $\mathrm{l}^{-1}, 0.02$ mol EDTA $\mathrm{l}^{-1}$ and 0.5 mol Tris- $\mathrm{HCl} \mathrm{l}^{-1}(\mathrm{pH} 8.5)$. Dithiothreitol $(1.65 \mathrm{~g})$ was added to the suspension with stirring under a nitrogen barrier. After incubation for $3 \mathrm{~h}$ at $37^{\circ} \mathrm{C}$, two volumes of cold ethanol were added to the mixture to precipitate DNA. The mixture was allowed to settle on ice for $1 \mathrm{~h}$ and was centrifuged at $12000 \mathrm{~g}$ for $15 \mathrm{~min}$ at $4^{\circ} \mathrm{C}$. A four-thirds volume of ice-cold ethanol was added to the supernatant with gentle stirring and the mixture was kept at $-20^{\circ} \mathrm{C}$ overnight, after which it was centrifuged at $12000 \mathrm{~g}$ for $17 \mathrm{~min}$ at $4^{\circ} \mathrm{C}$. The resulting precipitate was dried under vacuum. The precipitated sperm proteins were dissolved in $200 \mathrm{ml}$ buffer containing $0.2 \mathrm{~mol} \mathrm{NaCl} \mathrm{l^{-1 }}, 0.01 \mathrm{~mol} \mathrm{2-}$ mercaptoethanol $\mathrm{l}^{-1}, 4 \mathrm{~mol}$ urea $\mathrm{l}^{-1}$ and $0.05 \mathrm{~mol}$ sodium acetate $1^{-1}(\mathrm{pH} 5.3)$, and diluted with a buffer containing $0.01 \mathrm{~mol} 2$-mercaptoethanol $\mathrm{l}^{-1}$ and $0.01 \mathrm{~mol}$ sodium acetate $\mathrm{l}^{-1}$ ( $\mathrm{pH}$ 5.3). The mixture was applied to a CM-32 column $(1.2 \mathrm{~cm} \times 33.0 \mathrm{~cm}$; Whatman, Maidstone $)$ that had been equilibrated with a buffer containing $0.2 \mathrm{~mol} \mathrm{NaCl}^{-1}, 0.01$ mol 2-mercaptoethanol $\mathrm{l}^{-1}, 4 \mathrm{~mol}$ urea $\mathrm{l}^{-1}$ and $0.05 \mathrm{~mol}$ sodium acetate $1^{-1}(\mathrm{pH} 5.3)$. The proteins were eluted with a linear gradient of $0.2-1.0 \mathrm{~mol} \mathrm{NaCl}{ }^{-1}$ in a buffer containing 0.01 mol 2-mercaptoethanol $\mathrm{l}^{-1}, 4 \mathrm{~mol}$ urea $\mathrm{l}^{-1}$ and $0.05 \mathrm{~mol}$ sodium acetate $\mathrm{l}^{-1}(\mathrm{pH}$ 5.3). The eluted proteins were precipitated by addition of $80 \%(\mathrm{w} / \mathrm{v})$ trichloroacetic acid to a final concentration of $20 \%(\mathrm{w} / \mathrm{v})$. The precipitated proteins were dissolved in a buffer containing 0.7 mol 2-mercap-

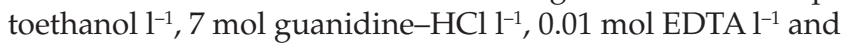
$0.05 \mathrm{~mol}$ Tris- $\mathrm{HCl} \mathrm{l}^{-1}(\mathrm{pH} 8.5)$. The $\mathrm{pH}$ of the solution was adjusted to 2.0 with $10 \%(\mathrm{v} / \mathrm{v})$ trifluoroacetic acid and the solution was incubated at $37^{\circ} \mathrm{C}$ for $15 \mathrm{~min}$. The proteins were purified by reverse-phase high-performance liquid chromatography on a Nucleosil 3007018 column (MachereyNagel, Düren) equilibrated in $0.1 \%(\mathrm{v} / \mathrm{v})$ trifluoroacetic acid. The proteins were eluted with a gradient of $0-80 \%(\mathrm{v} / \mathrm{v})$ acetonitrile in $0.1 \%(\mathrm{v} / \mathrm{v})$ trifluoroacetic acid at a flow rate of

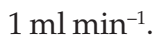

\section{Preparation of antiserum against boar sperm protamine}

Immunization and purification. Antiserum against boar protamine was raised in New Zealand White rabbits. Purified protamine was dissolved in Dulbecco's modified phosphate buffered saline (DPBS; Nissui Permaceutical Co Ltd, Tokyo) to a final concentration of $4 \mathrm{mg} \mathrm{ml}^{-1}$. The protamine solution was emulsified in an equal volume of Freund's complete adjuvant (Yatron, Tokyo). The animals were injected with $1 \mathrm{ml}$ of the emulsion intracutaneously at 2 week intervals and blood was collected through an ear vein 1 week after each injection. Serum was obtained and characterized with respect to titre using dot blotting as described by Hawkes et al. (1982).

The antiserum against protamine was affinity-purified using an $N$-hydroxysuccinimide-activated HiTrap column (Amersham Pharmacia Biotech, Uppsala) coupled with $2 \mathrm{mg}$ purified protamine $\mathrm{ml}^{-1}$. Saturated ammonium sulphate $(2.7 \mathrm{ml})$ and DPBS $(2 \mathrm{ml})$ were added to $2 \mathrm{ml}$ antiserum. After incubation for $1 \mathrm{~h}$ at $4^{\circ} \mathrm{C}$, the mixture was centrifuged at 
$10000 \mathrm{~g}$ for $30 \mathrm{~min}$ at $4^{\circ} \mathrm{C}$. The precipitate was dissolved in Tris-buffered saline (TBS; 0.05 mol Tris- $\mathrm{HCl} \mathrm{l}^{-1}(\mathrm{pH}$ 7.3)

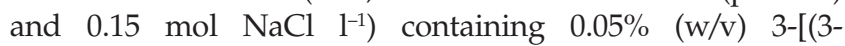
cholamidoproplyl)-dimethylammonio]-1-propanesulphonate (Chaps; Sigma Chemical Co, St Louis, MO) and applied to the protamine-coupled column. The column was incubated at room temperature for $1 \mathrm{~h}$, followed by three washes with TBS containing $0.05 \%(\mathrm{w} / \mathrm{v})$ Chaps. The antiserum against protamine was eluted from the column with $0.1 \mathrm{~mol}$ glycine- $\mathrm{HCl} \mathrm{l}^{-1}(\mathrm{pH}$ 2.0). The eluate was neutralized by adding $150 \mu \mathrm{l}$ of $2 \mathrm{~mol}$ Tris- $\mathrm{HCl} \mathrm{l}^{-1}$ ( $\mathrm{pH} 8.5$ ) to each fraction $(1.5 \mathrm{ml})$. The affinity-purified antiserum was stored at $-80^{\circ} \mathrm{C}$ until required.

Immunoblotting. The purified protamine $(1 \mu \mathrm{g})$ and histones ( $4 \mu \mathrm{g}$; Sigma Chemical Co) were separated by electrophoresis in acid-urea gel as described by Panyim and Chalkley (1969). Gels of $15 \%$ (w/v) acrylamide in $5.4 \mathrm{~mol}$ urea $1^{-1}$ and $0.9 \mathrm{~mol}$ acetic acid $\mathrm{l}^{-1}$ were made and pre-run at $200 \mathrm{~V}$ for $4 \mathrm{~h}$ using $0.9 \mathrm{~mol}$ acetic acid $\mathrm{l}^{-1}$ as a running buffer. The proteins were dissolved in $10 \mu \mathrm{l}$ of $7 \mathrm{~mol}$ urea $\mathrm{l}^{-1}$ and 0.9 mol acetic acid $\mathrm{l}^{-1}$, applied to the gel and electrophoresis was performed. The gel was soaked in the transfer buffer $(7 \%$ $(\mathrm{v} / \mathrm{v})$ acetic acid) for $10 \mathrm{~min}$, placed against a polyvinylidene difluoride membrane (Immobilon; Millipore Corporation, Bedford, MA) and the proteins were transferred using a transblot apparatus (MilliBlot; Millipore) operated according to the manufacturer's instructions. After transfer, the membrane was blocked in TBS with $0.5 \%(\mathrm{w} / \mathrm{v})$ casein (casein TBS) at room temperature for $0.5 \mathrm{~h}$. The membrane was incubated with the affinity-purified antiserum against protamine $(1: 100)$ overnight at $4^{\circ} \mathrm{C}$. After washing with TBS containing $0.05 \%(\mathrm{v} / \mathrm{v})$ Tween 20 , the membrane was incubated with peroxidase-labelled anti-rabbit immunoglobulin (Cappel Research Product, Durham, NC). A peroxidase immunostaining kit (Wako Pure Chemical Industries Ltd, Osaka) was used to visualize the immunoreaction.

Immunohistochemistry. Immunohistochemical analysis was performed as described by Noguchi et al. (1997), with slight modifications. Testes from an adult Landrace boar were fixed with Methacarn solution (methanol-chloroform-acetic acid, $6: 3: 1$, by volume) and embedded in paraffin wax. Sections were cut at $4 \mu \mathrm{m}$ thickness. These sections were deparaffinized, autoclaved at $121^{\circ} \mathrm{C}$ for $5-15 \mathrm{~min}$ in $0.1 \mathrm{~mol}$ citric acid $1^{-1}$ ( $\mathrm{pH}$ 6.0) for generation of antigen and incubated in $0.5 \%(\mathrm{w} / \mathrm{v})$ periodic acid to block endogenous peroxidase. After blocking with casein TBS, the sections were incubated overnight at $4^{\circ} \mathrm{C}$ with the affinity-purified antiserum against protamine at a dilution of $1: 80$ in casein TBS. The Elite ABC kit (Vector Laboratories Inc, Burlingame, CA) with diaminobenzidine was used to visualize the reaction. All the experiments were repeated at least twice.

The specificity of the antiserum was examined by testing whether immunopositive reactions were diminished by the use of immunoabsorbed antiserum, which was prepared as follows: the affinity-purified antiserum (1:40 dilution in TBS) was mixed with protamine to a final concentration of 2.5-25.0 $\mu \mathrm{g} \mathrm{ml}^{-1}$. After incubation at $37^{\circ} \mathrm{C}$ for $1 \mathrm{~h}$ and subsequently at $4^{\circ} \mathrm{C}$ overnight, the mixture was centrifuged at
$10000 \mathrm{~g}$ for $1 \mathrm{~h}$ at $4^{\circ} \mathrm{C}$. The supernatant was used for immunohistochemical staining at the same dilution as untreated antiserum.

\section{Detection of protamine in fertilized pig oocytes}

In vitro maturation and IVF of pig follicular oocytes. Pig ovaries were obtained from prepubertal gilts at a local abattoir and were transported to the laboratory at $35^{\circ} \mathrm{C}$. Cumulus-oocyte complexes (COCs) were collected from follicles 3-5 mm in diameter as described by Kikuchi et al. (1993, 1995). The COCs were transferred to a maturation medium (NCSU-37 solution; Petters and Wells, 1993) containing $10 \% \quad(\mathrm{v} / \mathrm{v})$ pig follicular fluid, $200 \mu \mathrm{mol}$ cysteamine $\mathrm{l}^{-1}, 2.5 \mu \mathrm{g} \mathrm{FSH} \mathrm{ml^{-1 }}$ (Sigma Chemical Co), $100 \mathrm{iu}$ penicillin $\mathrm{G}$ potassium $\mathrm{ml}^{-1}$ (Sigma) and $0.1 \mathrm{mg}$ streptomycin sulphate $\mathrm{ml}^{-1}$ (Sigma). About $40 \mathrm{COCs}$ were cultured for $46-48 \mathrm{~h}$ in $500 \mu \mathrm{l}$ maturation medium at $39^{\circ} \mathrm{C}$ under $5 \% \mathrm{CO}_{2}$ in air. The COCs were transferred to PBS containing $150 \mathrm{iu}$ hyaluronidase $\mathrm{ml}^{-1}$ (Sigma) for $5 \mathrm{~min}$ and freed from cumulus cells mechanically using a fine bore pipette. Denuded oocytes that emitted the first polar body were selected as matured oocytes. IVF was carried out as described by Kikuchi et al. (1995). Epididymal spermatozoa from a Landrace boar were frozen (Kikuchi et al., 1998). After thawing, spermatozoa $\left(5 \times 10^{8}\right.$ cells $\left.\mathrm{ml}^{-1}\right)$ were preincubated at $37^{\circ} \mathrm{C}$ for $30 \mathrm{~min}$ in TCM-199 (with Earl's salts; Gibco BRL, Life Technologies, Grand Island, NY) supplemented with $10 \%$ fetal bovine serum and antibiotics after adjusting the $\mathrm{pH}$ to 7.8 (Nagai et al., 1988). Preincubated spermatozoa were diluted once with Bracket and Oliphant solution (Bracket and Oliphant, 1975) supplemented with 5 mmol caffeine $\mathrm{l}^{-1}$ (Sigma) and $100 \mathrm{mg}$ casein phosphopeptide $\mathrm{ml}^{-1}$ (Meiji Seika Kaisha Ltd, Tokyo), and a portion of preincubated spermatozoa $(10 \mu \mathrm{l})$ was transferred to $90 \mu \mathrm{l}$ fertilization medium containing ten matured oocytes. The final sperm concentration was $2 \times 10^{5}$ cells $\mathrm{ml}^{-1}$. Co-incubation of oocytes with spermatozoa was carried out for $3.5 \mathrm{~h}$, and the oocytes were then transferred to BMOC-II solution (Brinster, 1965) containing $4 \mathrm{mg} \mathrm{BSA} \mathrm{ml}^{-1}$. The oocytes were subsequently cultured for $1.5 \mathrm{~h}$. At $1,2,3$ and $5 \mathrm{~h}$ after insemination the oocytes were freed from spermatozoa attached to the zona pellucida. Some oocytes were wholemounted on a glass slide, fixed in acetic alcohol (1:3), stained with $1 \%(\mathrm{w} / \mathrm{v})$ aceto-orcein solution and examined under a phase contrast microscope. The other oocytes were fixed in $3 \%(\mathrm{w} / \mathrm{v})$ paraformaldehyde and were processed for histological evaluation.

Immunohistochemical evaluation of protamine in penetrated sperm nuclei. The fixed in vitro-matured and in vitro-fertilized oocytes were embedded in $4 \%(\mathrm{w} / \mathrm{v})$ agar (Difco Laboratories, Detroit, MI); the agar was dehydrated and embedded in paraffin. Serial sections were cut at $3 \mu \mathrm{m}$ thickness. After deparaffinization, oocytes fixed at $1-5 \mathrm{~h}$ after insemination were processed for immunohistochemical analysis. The procedure for immunohistochemical analysis of oocytes was basically the same as described above, except that the oocytes were autoclaved for $5 \mathrm{~min}$ and blocked 
with $1.5 \%$ normal goat serum (Vector Laboratories). After immunostaining, the sections were counterstained with haematoxylin. Morphological changes of penetrating sperm nuclei and immunoreactivity with anti-protamine antiserum were evaluated.

Artificially induced decondensing sperm nuclei were evaluated following the same procedure as described for the fertilized oocytes to assess the immunoreactivity of decondensing sperm nuclei with anti-protamine antiserum. Frozen-thawed epididymal spermatozoa were incubated in DPBS containing $5 \mathrm{mmol}$ dithiothreitol $\mathrm{l}^{-1}$ for $30 \mathrm{~min}$ at $25^{\circ} \mathrm{C}$ $\left(1 \times 10^{7}\right.$ cells $\left.\mathrm{ml}^{-1}\right)$, fixed with paraformaldehyde, embedded in agar and immunohistochemical analysis was performed as described previously. Condensed sperm nuclei that had not undergone dithiothreitol treatment were also evaluated. Some of the sections reacted without the first antibody (anti-protamine antiserum) during immunohistochemical analysis. All sections were counterstained with haematoxylin after the immunoreaction.

Sequential changes of protamine dissociation during fertilization. The correlation between sperm nuclear changes in whole mount preparations and the status of protamine in serial sections were analysed to understand the sequential changes of protamine dissociation in fertilized oocytes. Firstly, the reliability of the data obtained by sectioning was determined by comparing morphological changes of penetrating sperm nuclei with those obtained by the whole mount method. Some sections containing oocytes at $5 \mathrm{~h}$ after insemination were incubated for $15 \mathrm{~min}$ in PBS containing $10 \mu \mathrm{g}$ Hoechst-33342 $\mathrm{ml}^{-1}$ (Hoechst; Calbiochem, CA) and the nuclear configuration of the penetrating sperm (condensation, decondensation and male pronuclei formation) was determined under a fluorescent microscope. The sperm nuclear status was recorded and reconstructed for each whole oocyte, and the results were compared with those for oocytes in the whole mount preparation. After this confirmation of data reliability, sequential changes in protamine dissociation were analysed.

\section{Statistical analysis}

Data were subjected to ANOVA using general linear model procedures (SAS/STAT, 1988). Morphological changes of penetrated sperm nuclei and immunohistochemical analysis of condensed sperm nuclei during IVF were analysed by Duncan's multiple range test after transformation using arcsin of percentages (Snedecor and Cochran, 1989).

\section{Results}

\section{Specificity of the antiserum against protamine}

The specificity of the antiserum for protamine was examined by acid-urea gel electrophoresis followed by western blotting and by immunohistochemical analysis (Figs 1 and 2, respectively). After transfer onto nitrocellulose membrane and staining with Coomassie brilliant blue, the protamine band was relatively weak compared with the bands for core histones (Fig. 1, lanes 1 and 2). When electrophoresis was followed by immunoblotting with the antiserum, a specific band for protamine was detected, but bands for somatic histones were not detected (Fig. 1, lanes 3 and 4). Immunohistochemical evaluation of boar testis showed specific staining in nuclei of fully elongated spermatids, but no reaction in other cells, such as round spermatids, spermatocytes and spermatogonias (Fig. 2a). The immunohistochemical analysis was repeated using antiserum preincubated with purified protamine (immunoabsorbed antiserum) to confirm the specificity of the antiserum for protamine. No immunoreaction was observed after immunohistochemical analysis with the preincubated antiserum (Fig. 2b).

\section{Sequential changes of penetrating sperm nuclei}

Sequential changes of penetrating sperm nuclei were observed in whole mount preparations of inseminated oocytes. The rates of sperm penetration, polyspermy and the number of spermatozoa per oocyte are shown (Fig. 3). Sperm penetration was observed in $>30 \%$ of oocytes at $2 \mathrm{~h}$ after insemination. At $3-5 \mathrm{~h}$ after insemination, sperm penetration was observed in almost all oocytes. The incidence of polyspermic penetration and the mean number of penetrated spermatozoa per oocyte increased with increasing duration of insemination. Penetrating sperm nuclei were classified as condensed sperm nuclei,

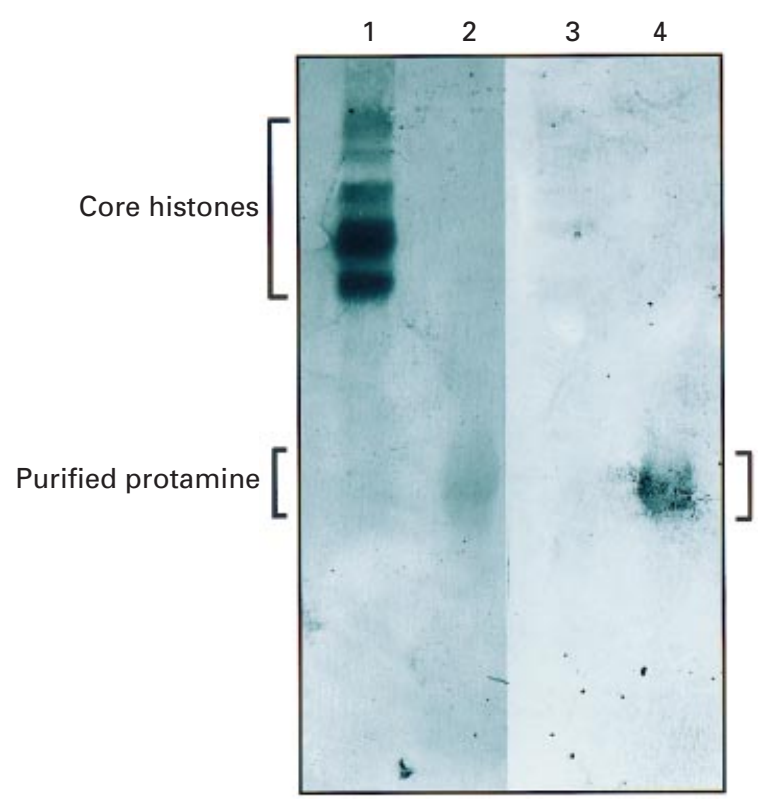

Fig. 1. Detection of core histones and protamine. Core histones (lanes 1 and 3) and purified protamine (lanes 2 and 4) were run on an acid-urea gel, transferred to nitrocellulose membrane and stained with Coomassie brilliant blue (lanes 1 and 2) or immunostained with the anti-protamine antiserum (lanes 3 and 4). Both core histones and purified protamine stained with Coomassie brilliant blue, whereas purified protamine but not core histone was detected by immunostaining. 

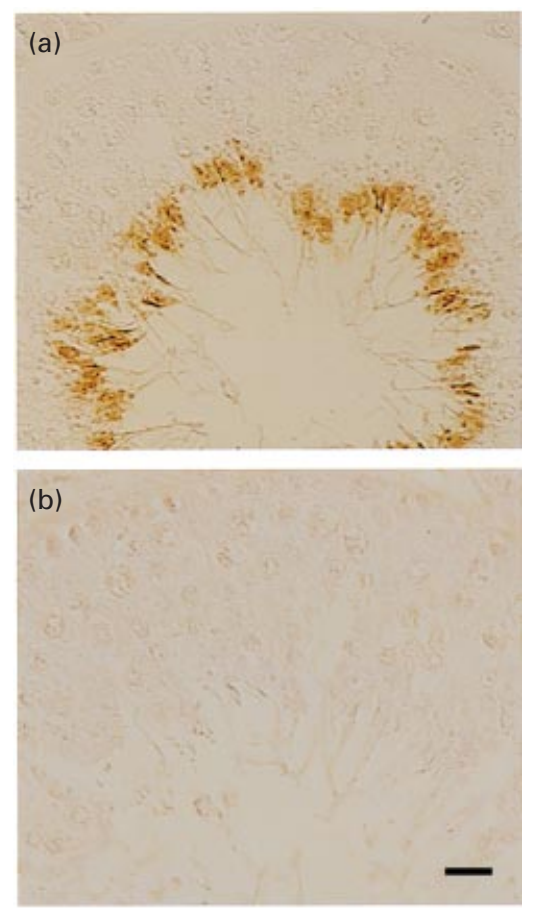

Fig. 2. Boar testis stained using (a) purified anti-protamine antiserum and (b) immunoabsorbed antiserum. Note that nuclei of fully elongated spermatids were positive, but not other cells such as round spermatids, spermatocytes and spermatogonia. The positive reaction in the fully elongated spermatids disappeared when the sections were treated with absorbed antiserum. Scale bar represents $10 \mu \mathrm{m}$.

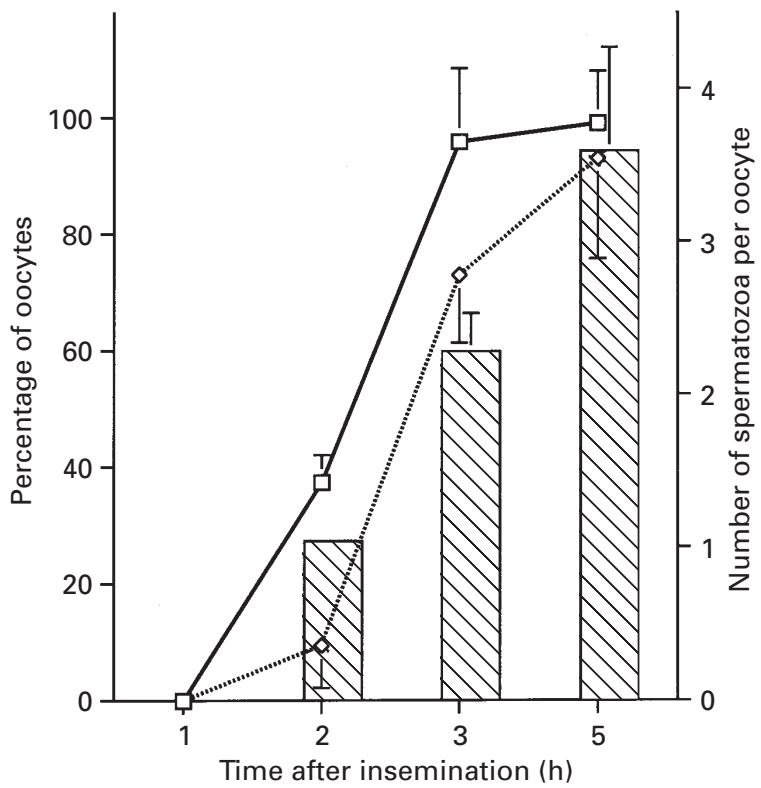

Fig. 3. Rate of sperm penetration ( $\square)$, rate of polyspermy $(\diamond)$ and sperm number per oocyte $(\mathbb{\mathbb { N }})$ of pig oocytes fertilized in vitro. Oocytes matured in vitro were inseminated and fixed in whole mount preparation at $1,2,3$ and $5 \mathrm{~h}$ after insemination. The oocytes were stained with $1 \%(\mathrm{w} / \mathrm{v})$ aceto-orcein and examined under a phase-contrast microscope.
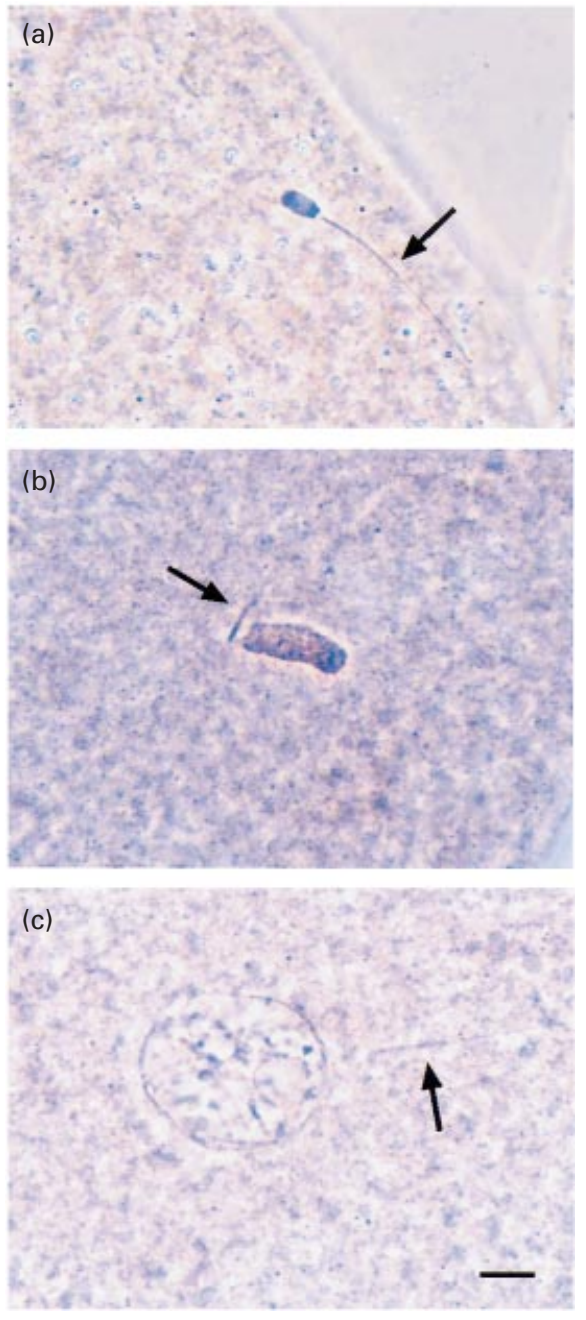

Fig. 4. Morphological changes of penetrating boar sperm nuclei in whole mount preparations. (a) A condensed sperm nucleus at $2 \mathrm{~h}$ after insemination, (b) a decondensing sperm nucleus $5 \mathrm{~h}$ after insemination and (c) a male pronucleus $5 \mathrm{~h}$ after insemination. Arrows indicate sperm tails associated with sperm nuclei. Scale bar represents $10 \mu \mathrm{m}$.

decondensing sperm nuclei and male pronuclei (Fig. 4a,b,c). Sequential changes in sperm nuclei after insemination are shown (Table 1). At $2 \mathrm{~h}$ after insemination, all sperm nuclei were in a condensed state. Decondensing spermatozoa were first observed at $3 \mathrm{~h}$ after insemination, and male pronuclei were first observed at $5 \mathrm{~h}$ after insemination. The condensed sperm nuclei changed to decondensing nuclei and then to male pronuclei as the duration of the insemination period was increased.

\section{Protamine status in penetrated sperm nuclei}

Examination of sectioned oocytes under light microscopy enabled all condensed sperm nuclei and some of the decondensing sperm nuclei to be observed. Immuno- 
Table 1. Morphological changes in penetrating sperm nuclei during in vitro fertilization of pig oocytes

\begin{tabular}{|c|c|c|c|c|}
\hline \multirow{2}{*}{$\begin{array}{l}\text { Culture period (h } \\
\text { after insemination) }\end{array}$} & \multirow{2}{*}{$\begin{array}{l}\text { Total number of } \\
\text { sperm nuclei } \\
\text { examined }\end{array}$} & \multicolumn{3}{|c|}{ Number (and \%) of penetrating sperm nuclei } \\
\hline & & Condensed & Decondensing & Male pronuclei \\
\hline 2 & 31 & $31(100 \pm 0.0)^{\mathrm{a}}$ & $0(0 \pm 0.0)^{\mathrm{a}}$ & $0(0 \pm 0.0)^{\mathrm{a}}$ \\
\hline 3 & 161 & $104(65 \pm 4.8)^{\mathrm{b}}$ & $57(35 \pm 4.0)^{\mathrm{b}}$ & $0(0 \pm 0.0)^{a}$ \\
\hline 5 & 258 & $37(14 \pm 3.6)^{c}$ & $95(37 \pm 3.6)^{b}$ & $126(49 \pm 4.6)^{b}$ \\
\hline
\end{tabular}

Three replicate trials were performed.

Values are mean number (percentage \pm SEM).

Inseminated oocytes were examined in whole mount preparations.

${ }^{\mathrm{abc}}$ Different superscripts within a column indicate significant differences between percentages by Duncan's multiple-range test $(P<0.05)$.

histochemical reactivity in the condensed sperm nuclei after penetration is shown (Fig. 5). Three types of sperm nuclei with or without immunoreactivity were detected (Fig. 5a,b). The three types of sperm nuclei were: (i) protamine associated with nucleus; the diaminobenzidine signal was observed all over the sperm nuclei and haematoxylin staining was not visible; (ii) protamine partially dissociated from the nucleus; part of the area of sperm nuclei was stained
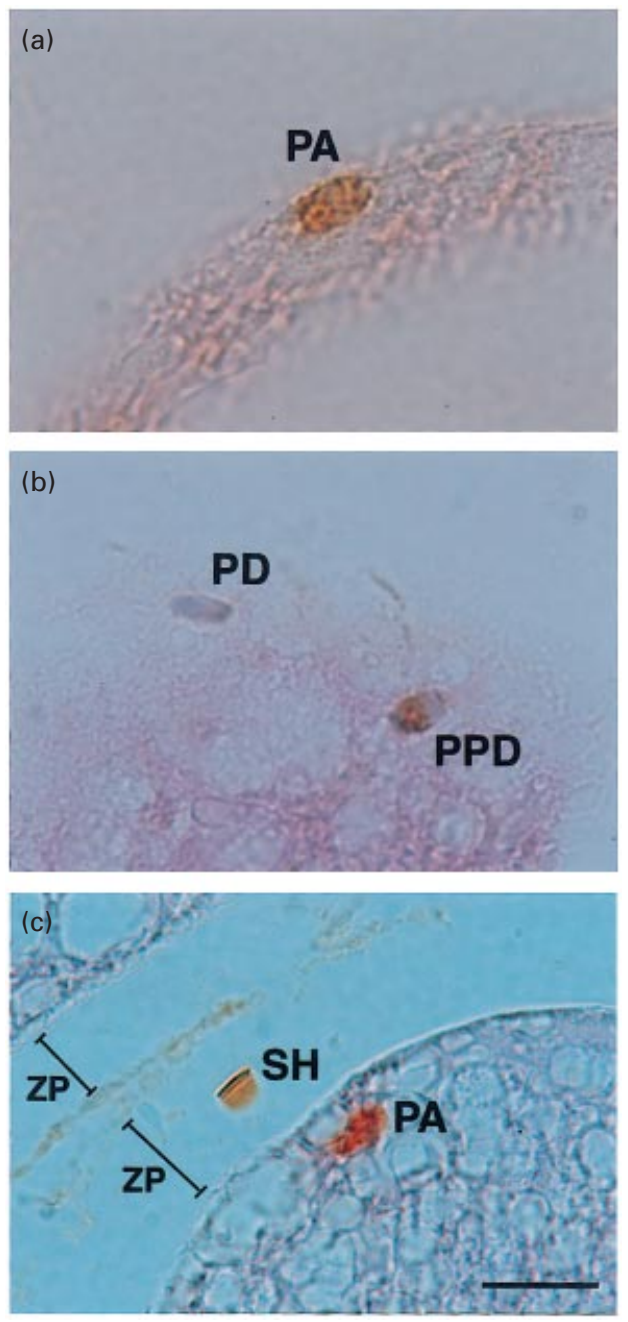

with diaminobenzidine and the remaining area was stained with haematoxylin; and (iii) protamine fully dissociated from the nucleus; no diaminobenzidine signal was observed and sperm nuclei were stained with haematoxylin only. Sequential changes of immunoreactivity in condensed sperm nuclei during fertilization are shown (Table 2). At $2 \mathrm{~h}$ after insemination, $>90 \%$ of condensed sperm nuclei were classified as 'protamine associated with nucleus', and no sperm nuclei were classified as 'protamine fully dissociated from the nucleus'. At $3 \mathrm{~h}$ after insemination, the incidence of sperm nuclei classified as 'protamine associated with nucleus' compared with the condensed spermatozoa decreased significantly $(P<0.05)$ and about half of the sperm nuclei were determined as 'protamine fully dissociated from the nucleus'. At $5 \mathrm{~h}$ after insemination, the incidence of sperm nuclei classified as 'protamine partially dissociated from the nucleus' and as 'protamine fully dissociated from the nucleus' decreased significantly $(P<0.05)$ and additional sperm penetration (an increase in the incidence of sperm nuclei classified as 'protamine associated with nucleus' compared with at $3 \mathrm{~h}$ after insemination) was observed. Decondensing sperm nuclei showed no staining with diaminobenzidine, but stained to varying degrees with haematoxylin (data not shown).

As decondensing sperm nuclei were not stained with diaminobenzidine, the question arose as to whether antibody binding to retained protamine was not observed because decondensing chromatin was very dispersed. Sperm nuclei

Fig. 5. Immunohistochemical analysis of penetrating boar sperm nuclei in oocytes, using anti-protamine antiserum. ( $\mathrm{a}, \mathrm{b}$ and $\mathrm{c}$ ) Paraffin wax sections of oocytes at $3 \mathrm{~h}$ after insemination were examined by immunohistochemistry and counterstained with haematoxylin. Penetrated sperm nuclei were classified into three groups according to immunoreactivity: (i) protamine associated with nucleus (PA): the diaminobenzidine signal was observed all over the sperm nuclei and haematoxylin staining was not visible; (ii) protamine partially dissociated from nucleus (PPD): part of the area of sperm nuclei was stained with diaminobenzidine and the remaining area was stained with haematoxylin; and (iii) protamine fully dissociated from nucleus (PD); no diaminobenzidine signal was observed and sperm nuclei were stained with haematoxylin only. Sperm head (SH) before penetration located in the zona pellucida (ZP) was also immunoreactive. Scale bar represents $10 \mu \mathrm{m}$. 
Table 2. Protamine immunohistochemical analysis in condensed sperm nuclei during in vitro fertilization of pig oocytes

\begin{tabular}{|c|c|c|c|c|}
\hline \multirow[b]{2}{*}{$\begin{array}{l}\text { Culture period (h } \\
\text { after insemination) }\end{array}$} & \multirow{2}{*}{$\begin{array}{l}\text { Total number of } \\
\text { condensed sperm } \\
\text { nuclei examined }\end{array}$} & \multicolumn{3}{|c|}{ Number of condensed sperm nuclei } \\
\hline & & $\begin{array}{c}\text { Protamine } \\
\text { associated (\%) }\end{array}$ & $\begin{array}{l}\text { Protamine partially } \\
\text { dissociated }(\%)\end{array}$ & $\begin{array}{l}\text { Protamine fully } \\
\text { dissociated (\%) }\end{array}$ \\
\hline 2 & 51 & $47(87 \pm 19.4)^{\mathrm{a}}$ & $7(13 \pm 5.9)^{\mathrm{ab}}$ & $0(0 \pm 0.0)^{\mathrm{a}}$ \\
\hline 3 & 199 & $60(30 \pm 4.8)^{b}$ & $44(22 \pm 1.9)^{\mathrm{b}}$ & $95(48 \pm 7.1)^{\mathrm{b}}$ \\
\hline 5 & 69 & $61(88 \pm 11.3)^{a}$ & $4(6 \pm 1.5)^{a}$ & $4(6 \pm 1.5)^{\mathrm{a}}$ \\
\hline
\end{tabular}

Values are mean number (percentage \pm SEM).

Condensed sperm nuclei: serial sections of fertilized oocytes were examined immunohistochemically using anti-protamine antiserum reactivity of condensed sperm nuclei in the oocytes.

Protamine associated: the immunoreaction with anti-protamine antiserum was observed all over the sperm nuclei and haematoxylin staining was not visible.

Protamine partially dissociated: part of the area of sperm nuclei was immunoreacted and the remaining area was stained with haematoxylin.

Protamine fully dissociated: no immunoreaction was observed and sperm nuclei stained only with haematoxylin.

abc Different superscripts within a column indicate significant differences between percentages by Duncan's multiple-range test $(P<0.05)$.

were made to decondense in vitro and were evaluated with the same procedure as for fertilized oocytes to eliminate this possibility. After incubation for $30 \mathrm{~min}$ with dithiothreitol, sperm nuclei decondensed randomly. In vitro decondensed sperm nuclei (Fig. 6c) and condensed sperm nuclei without dithiothreitol treatment (Fig. 6a) could be stained with anti-protamine antiserum. Both condensed and decondensed sperm nuclei were not stained with diaminobenzidine when the immunoreaction was performed without the antibody (Fig. 6b,d). These results also show that staining of decondensed sperm nuclei with haematoxylin was lower than that of condensed sperm nuclei. These results indicate clearly that the lack of staining with diaminobenzidine in decondensing sperm nuclei during fertilization is not caused by dispersion of chromatin.

\section{Protamine dissociation from sperm nuclei during fertilization}

It was difficult to detect all decondensing sperm nuclei and male pronuclei by haematoxylin staining during immunohistochemical analysis because of their low staining. The data for morphological changes obtained by the whole mounted method should be used to confirm the relationship between morphological changes and the dynamics of protamine in penetrated sperm nuclei. The reliability of the sectioning method was checked earlier by staining with Hoechst and comparing the results with those obtained by the whole mounted method. Hoechst staining enables decondensing sperm nuclei and male pronuclei to be visualized, as well as condensed sperm nuclei (Fig. 7). The incidence of each of these categories in sectioned oocytes stained with Hoechst was not different from those in whole mounted samples (Table 3). Thus, we are able to compare the results from immunohistochemical analysis (Table 2) with those from the whole mount method (Table 1), showing the relationship between morphological changes and the dynamics of protamine in penetrated sperm nuclei (Fig. 8). This analysis shows clearly that the incidence of sperm nuclei classified as 'protamine associated with nucleus' decreased as the duration of the insemination period was increased.

\section{Discussion}

In the present study, the relationship between protamine dissociation and morphological changes of sperm nuclei during fertilization was investigated. For this purpose, boar protamine was purified by high-performance liquid chromatography, polyclonal antiserum against boar protamine was raised and further affinity-purified with purified protamine. The antiserum reacted exclusively with boar protamine on western blotting and there was no crossreactivity with core histones. Furthermore, indirect immunohistochemical examination of testes using the antiserum revealed that only fully developed spermatid heads stained strongly. This specificity has been observed not only in pigs but also in other mammalian species (rats and mice; J. Noguchi, A. Shimada, K. Kikuchi and $\mathrm{H}$. Kaneko, unpublished). Rodman et al. (1981) and Nonchev and Tsanev (1990) reported that antibodies against spermspecific protein or protamine of mice also reacted with proteins of the female pronucleus in fertilized mouse oocytes. In contrast, no component other than condensed boar sperm nuclei was apparently stained with antiprotamine antibody in the present study (data not shown). These results indicate that the antiserum developed in the present study is quite specific and, thus, should be a powerful tool for investigating the dynamics of protamine during fertilization.

The present results, based on immunohistochemical analysis, indicate that protamine dissociates from sperm nuclei before decondensation. These results also indicate that there are condensed sperm nuclei lacking protamine in pig oocytes 
Table 3. Comparison of pig oocyte examination methods

\begin{tabular}{lccccc}
\hline \multirow{2}{*}{$\begin{array}{l}\text { Oocyte } \\
\text { examination }\end{array}$} & $\begin{array}{c}\text { Total number } \\
\text { of oocytes } \\
\text { examined }\end{array}$ & $\begin{array}{c}\text { Total number } \\
\text { of sperm that } \\
\text { penetrated }\end{array}$ & Condensed (\%) & Decondensing (\%) & $\begin{array}{c}\text { Pronucleus } \\
\text { formed* }(\%)\end{array}$ \\
\hline $\begin{array}{l}\text { Mounted whole } \\
\text { Sectioned }\end{array}$ & 186 & 220 & $86(39)$ & $90(41)$ & $44(20)$ \\
\hline
\end{tabular}

All oocytes were collected and fixed at $5 \mathrm{~h}$ after insemination.

Sectioned: stained with Hoechst 33342 and examined under a fluorescent microscope.

*One of the pronuclei was not included in the data analysis as it was a female pronucleus.
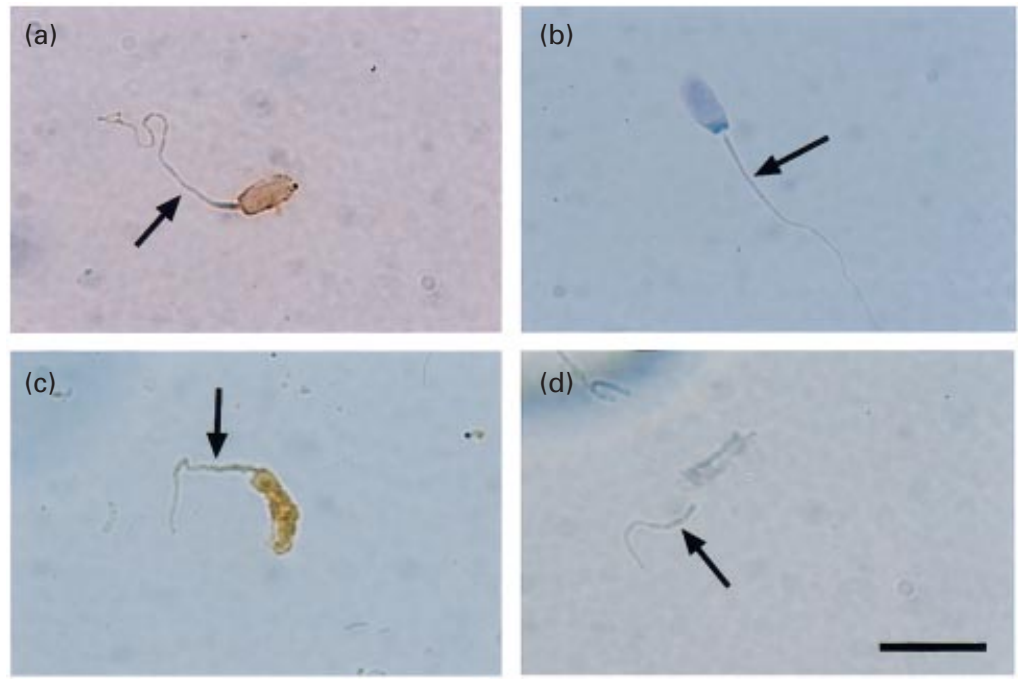

Fig. 6. Immunoreactivity of artificially induced decondensing boar sperm nuclei with anti-protamine antiserum. ( $a$ and $b$ ) Condensed sperm nuclei were evaluated without dithiothreitol treatment. (c and d) Frozen-thawed epididymal spermatozoa were treated with dithiothreitol for $30 \mathrm{~min}$, fixed with paraformaldehyde, embedded in agar and evaluated by immunohistochemical analysis following the same protocol as for fertilized oocytes. Some of the sections reacted without the first antibody (anti-protamine antiserum) during immunohistochemistry (b and $d$ ). All sections were counterstained with haematoxylin after the immunoreaction. Arrows indicate sperm tails associated with sperm nuclei. Scale bar represents $10 \mu \mathrm{m}$.

penetrated by a spermatozoon or spermatozoa. In toads, sperm nuclei lose protamines within $1 \mathrm{~min}$ after penetration, accompanied by nuclear decondensation (Ohsumi and Katagiri, 1991), indicating that removal of protamines occurs simultaneously with decondensation of nuclei during pronucleus formation (Katagiri and Ohsumi, 1994). However, hamster sperm nuclei microinjected into oocytes required 45-60 min for decondensation (Perreault et al., 1987). Taking our results into consideration, it is possible that removal of protamine is not sufficient for the decondensation of mammalian sperm nuclei, and that an unknown factor (or factors) other than protamine may participate in maintenance of the condensed state in the sperm nuclei. Rodman et al. (1981) reported that, in mice, sperm basic nuclear proteins are retained in decondensed sperm nuclei. Nonchev and Tsanev (1990) reported that protamine is retained in the male pronucleus before the onset of DNA replication. The reasons for this discrepancy in the timing of protamine disappearance among species are unclear. Spermatozoa from many species, including boars, contain only a single type of protamine, called protamine 1 (Balhorn, 1989). However, there are two different protamines, protamine 1 and protamine 2, in mice (Bellve et al., 1988). This variety of protamines may mean that, in mice, protamines are remodelled through a pathway different from that in pigs or other mammals.

Perreault et al. (1988) and Zirkin et al., (1989) suggested that reduction of protamine S-S is required for decondensation of sperm nuclei in vivo, and Katagiri and Ohsumi (1994) suggested that protamine is reduced by glutathione. Furthermore, pronuclear eggs lack sufficient reducing power to effect sperm nuclear decondensation (Perreault et al., 1984), and mature oocytes contain significantly more glutathione than do pronuclear eggs (Perreault et al., 1988). At $2-3 \mathrm{~h}$ after insemination the incidence of protamine-dissociated nuclei 

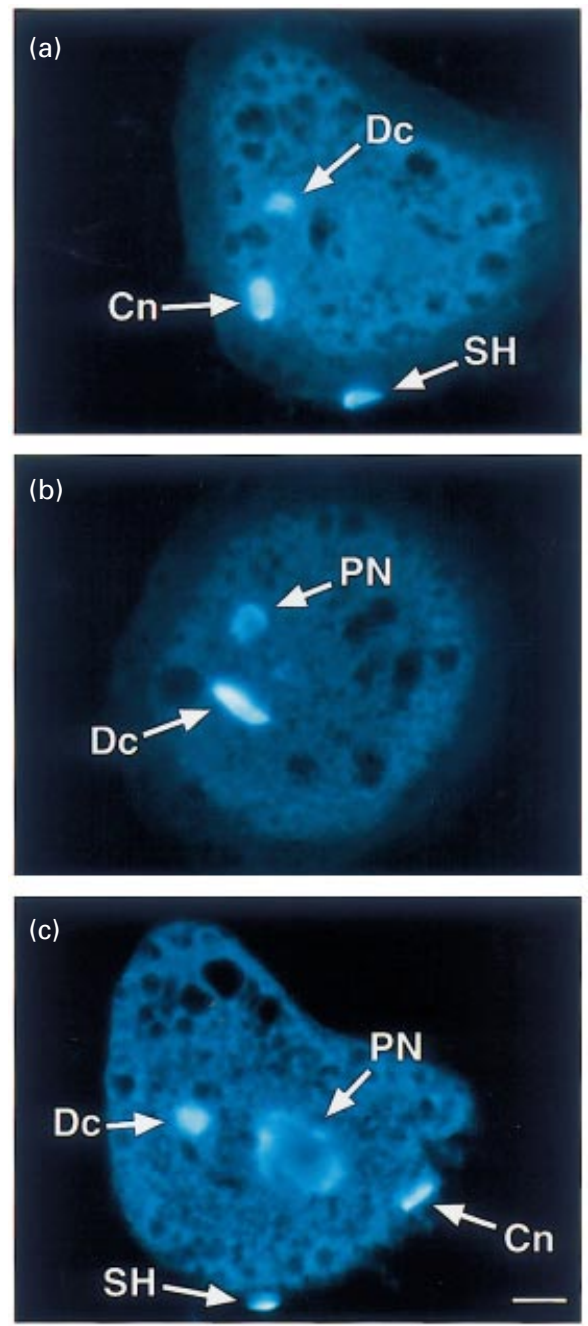

Fig. 7. Morphological changes in penetrating boar sperm nuclei after histological preparation and staining with Hoechst 33342 dye. ( $\mathrm{a}, \mathrm{b}$ and $\mathrm{c}$ ) Examples of oocytes fixed at $5 \mathrm{~h}$ after insemination. Condensed (Cn) and decondensing (Dc) sperm nuclei and pronuclei $(\mathrm{PN})$ are visible. Sperm heads $(\mathrm{SH})$ before penetration, attached to the zona pellucida, are also visible. Scale bar represents $10 \mu \mathrm{m}$.

(both partially and fully dissociated) was increased. However, at $5 \mathrm{~h}$ after insemination, almost all the condensed sperm nuclei, which were derived from delayed polyspermic penetration, were found in association with protamine. Thus, at $3-5 \mathrm{~h}$ after insemination, protamine is rarely dissociated from the penetrating sperm nuclei. This inability to bring about dissociation of protamine is probably caused by a low concentration of glutathione in the polyspermic oocyte at $5 \mathrm{~h}$ after insemination. In fact, the glutathione concentration decreases in fertilized pig oocytes by $6 \mathrm{~h}$ after insemination (Funahashi et al., 1995). It is possible that reduction of protamine by cytoplasmic glutathione is a prerequisite for protamine dissociation from sperm condensed nuclei.

In mammals, little is known about the oocyte factors that are responsible for protamine removal and nuclear

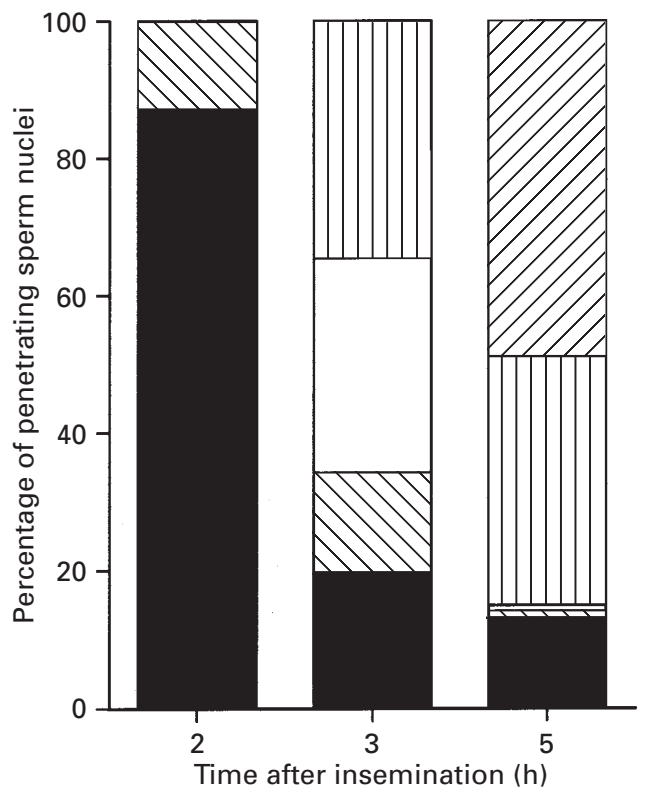

Fig. 8. Protamine dissociation from boar sperm nuclei before decondensation. 遂: Pronuclei. 四: Decondensing sperm nuclei. $\square$ : Protamine fully dissociated from the nucleus. $\mathbb{\mathbb { N }}$ : Protamine partially dissociated from the nucleus. 口: Protamine associated with the nucleus. These last three categories all represent condensed nuclei.

decondensation during fertilization (Zirkin et al., 1989). Recent studies have revealed that nucleoplasmin plays a major role in the removal of sperm-specific protein in amphibians (Ohsumi and Katagiri, 1991; Philpot et al., 1991; Philpot and Leno, 1992). Although the protamine releasing factors have not been identified in mammals, human spermatozoa treated with dithiothreitol are decondensed in Xenopus egg extracts (Brown et al., 1987; Ohsumi et al., 1988). Furthermore, nucleoplasmin purified from Bufo egg extract selectively removes protamines from human sperm nuclei in vitro (Itoh et al., 1993). These studies indicate that the mammalian egg cytoplasm contain factors, similar to amphibian nucleoplasmin, that participates in the removal of protamine. Further studies are needed to elucidate the mechanisms by which protamine is removed from sperm nuclei in the cytoplasm of mammalian eggs.

In conclusion, a specific antiserum was raised against boar protamine and, by using this antiserum, it was found that protamine dissociates from sperm nuclei before decondensation during fertilization of pig oocytes.

This study was supported by a Domestic Research Fellowship from the Japan Science and Technology Corporation. The authors would like to thank T. Nagai for critical reading of the manuscript, D.A. Vaughan for advice about the English and T. Aoki for technical assistance.

\section{References}

Akama K, Oka S, Tobita T and Hayashi H (1989) Changes in chromatin structure of boar late spermatids to mature spermatozoa by using modification with dansyl chloride Biochimica et Biophysica Acta 1008 198-202 
Balhorn R (1989) Mammalian protamines: structure and molecular interactions. In Molecular Biology of Chromosome Function pp 366-395 Ed. KW Adolph. Springer Verlag, New York

Bedford JM and Calvin HI (1974) The occurrence and possible functional significance of -S-S- crosslinks in sperm heads, with particular reference to eutherian mammals Journal of Experimental Zoology 188 137-156

Bellve AR, McKay DJ, Renaux BS and Dixon GH (1988) Purification and characterization of mouse protamines P1 and P2: amino acid sequence of P2 Biochemistry 27 2890-2897

Bracket BG and Oliphant G (1975) Capacitation of rabbit spermatozoa in vitro. Biology of Reproduction 12 260-274

Brinster RL (1965) Studies on the development of mouse embryos in vitro. IV. Interaction of energy source Journal of Reproduction and Fertility 10 227-240

Brown DB, Blake EJ, Wolgemuth DJ, Gordon K and Ruddle FH (1987) Chromatin decondensation and DNA synthesis in human sperm activated in vitro by using Xenopus laevis egg extracts Journal of Experimental Zoology 242 215-231

Calvin HI and Bedford JM (1971) Formation of disulfide bonds in the nucleus and accessory structures of mammalian spermatozoa during maturation in the epididymis Journal of Reproduction and Fertility Supplement 13 65-75

Calvin HI, Yu CC and Bedford JM (1973) Effects of epididymal maturation, zinc (II) and copper (II) on the reactive sulfhydryl content of structural elements in rat spermatozoa Experimental Cell Research 81 333-341

Ecklund PS and Levine L (1975) Mouse sperm nuclear basic protein. Electrophoretic characterization and fate after fertilization Journal of Cell Biology 66 251-262

Funahashi H, Stumpf TT, Cantley TC, Kim NH and Day BN (1995) Pronuclear formation and intracellular glutathione content of in vitromaturated porcine oocytes following in vitro fertilization and/or electrical activation Zygote 3 273-281

Hawkes R, Niday E and Gordon J (1982) A dot-immunobinding assay for monoclonal and other antibodies Analytical Biochemistry 119 142-147

Itoh T, Ohsumi K and Katagiri C (1993) Remodeling of human sperm chromatin mediated by nucleoplasmin from amphibian eggs Development Growth and Differentiation 35 59-66

Katagiri C and Ohsumi K (1994) Remodeling of sperm chromatin induced in egg extracts of amphibians International Journal of Developmental Biology 38 209-216

Kikuchi K, Nagai T, Motlik J, Shioya Y and Izaike Y (1993) Effect of follicle cells on in vitro fertilization of pig follicular oocytes Theriogenology 39 593-599

Kikuchi K, Naito K, Dean FP, Izaike Y and Toyoda Y (1995) Histone H1 kinase activity during in vitro fertilization of pig follicular oocytes matured in vitro. Theriogenology 43 523-532

Kikuchi K, Nagai T, Kashiwazaki N, Ikeda H, Noguchi J, Shimada A, Soloy E and Kaneko H (1998) Cryopreservation and ensuing in vitro fertilization ability of boar spermatozoa from epididymides stored at $4^{\circ} \mathrm{C}$ Theriogenology $50615-623$

Kopecny V and Pavlok A (1975) Autoradiographic study of mouse spermatozoa arginine-rich nuclear protein in fertilization Journal of Experimental Zoology 191 85-96

Marushige Y and Marushige K (1975) Transformation of sperm histone during formation and maturation of rat spermatozoa Journal of Biological Chemistry 250 39-45

Meistrich ML, Reid BO and Barcellona WJ (1976) Changes in sperm nuclei during spermatogenesis and epididymal maturation Experimental Cell Research 99 72-78

Nagai T, Takahashi T, Masuda H, Shioya Y, Kuwayama M, Fukushima M, Iwasaki S and Hanada A (1988) In-vitro fertilization of pig oocytes by frozen boar spermatozoa Journal of Reproduction and Fertility 84 585-591

Noguchi J, Hikono H, Sato S, Watanabe G, Taya K, Sasamoto S and Hasegawa Y (1997) Ontogeny of inhibin secretion in the rat testis: secretion of inhibin-related proteins from fetal Leydig cells and of bioactive inhibin from Sertoli cells Journal of Endocrinology 155 27-34

Nonchev S and Tsanev R (1990) Protamine-histone replacement and DNA replication in the male mouse pronucleus Molecular Reproduction and Development $2572-76$

Ohsumi K and Katagiri C (1991) Characterization of the ooplasmic factor inducing decondensation of and protamine removal from toad sperm nuclei: involvement of nucleoplasmin Developmental Biology 148 295-305

Ohsumi K, Katagiri C and Yanagimachi R (1988) Human sperm nuclei can transform into condensed chromosomes in Xenopus egg extracts Gamete Research 201-9

Panyim S and Chalkley G (1969) High resolution acrylamide gel electrophoresis of histones Archives of Biochemistry and Biophysics 130 337-346

Perreault SD (1992) Chromatin remodeling in mammalian zygotes Mutation Research 296 43-55

Perreault SD, Wolff RA and Zirkin BR (1984) The role of disulfide bond reduction during mammalian sperm nuclear decondensation in vivo. Developmental Biology 101 160-167

Perreault SD, Naish SJ and Zirkin BR (1987) The timing of hamster sperm nuclear decondensation and male pronucleus formation is related to sperm nuclear disulfide bond content Biology of Reproduction 36 239-244

Perreault SD, Barbee RR and Slott VL (1988) Importance of glutathione in the acquisition and maintenance of sperm nuclear decondensing activity in maturing hamster oocytes Developmental Biology 125 181-186

Petters RM and Wells KD (1993) Culture of pig embryos Journal of Reproduction and Fertility Supplement 48 61-73

Philpot A and Leno GH (1992) Nucleoplasmin remodels sperm chromatin in Xenopus egg extracts Cell 69 759-767

Philpot A, Leno GH and Laskey RA (1991) Sperm decondensation in Xenopus egg cytoplasm is mediated by nucleoplasmin Cell 65 569-578

Poccia D (1986) Remodeling of nucleoproteins during gametogenesis, fertilization, and early development International Reviews of Cytology 105 $1-65$

Rodman TC, Litwin SD, Romai M and Vidali G (1979) Life history of mouse sperm protein. Intratesticular stages Journal of Cell Biology 80 605-620

Rodman TC, Pruslin FH, Hoffman HP and Allfrey VG (1981) Turnover of basic chromosomal proteins in fertilized eggs: a cytoimmunochemical study of events in vivo. Journal of Cell Biology 90 351-361

Roux C, Gusse M, Chevaillier P and Dadoune JP (1988) An antiserum against protamines for immunohistochemical studies of histone to protamine transition during human spermatogenesis Journal of Reproduction and Fertility 82 35-42

SAS/STAT User's Guide (1988) Release Edition 6.03 pp 549-640. SAS Institute Inc., Cary, NC

Snedecor GW and Cochran WG (1989) Statistical Methods 8th Edn pp 273-296. Iowa State University Press, Iowa

Stanker LH, Wyrobek A, McKeown C and Balhorn R (1993) Identification of the binding site of two monoclonal antibodies to human protamine Molecular Immunology 30 1633-1638

Tobita T, Nomoto M, Nakano M and Ando T (1982) Isolation and characterization of nuclear basic protein (protamine) from boar spermatozoa Biochimica et Biophysica Acta 707 252-258

Tobita T, Tsutsumi H, Kato A, Suzuki H, Nomoto M, Nakano M and Ando T (1983) Complete amino acid sequence of boar protamine Biochimica et Biophysica Acta 744 141-146

Tobita T, Tanaka H, Tanaka K, Tanaka T, Kojima S and Nakano M (1984) Characteristics of cysteinyl sulfhydryls and nuclease sensitivity of boar sperm nuclei during epididymal maturation Biochemistry International 9 161-168

Yanagimachi R (1994) Mammalian fertilization. In The Physiology of Reproduction pp 189-317 Eds E Knobil and JD Neill. Raven Press Ltd, New York

Yoshida M (1993) Role of glutathione in the maturation and fertilization of pig oocytes in vitro. Molecular Reproduction and Development 35 76-81

Yoshida M, Ishigaki K, Nagai T, Chikyu M and Pursel VG (1993) Glutathione concentration during maturation and after fertilization in pig oocytes: relevance to the ability of oocytes to form male pronucleus Biology of Reproduction 49 89-94

Zirkin BR, Perreault SD and Naish SJ (1989) Formation and function of the male pronucleus during mammalian fertilization. In The Molecular Biology of Fertilization pp 91-114 Eds H Schatten and G Schatten. Academic Press, San Diego 